University of Wollongong

Research Online

Faculty of Engineering and Information

Faculty of Engineering and Information

Sciences - Papers: Part A

Sciences

2014

\title{
18F-FMISO PET/CT visualization of tumor hypoxia in patients with chordoma of the mobile and sacrococcygeal spine
}

\author{
Matthew D. Cheney \\ Harvard Medical School, Boston \\ Yen-Lin Chen \\ Massachusetts General Hospital \\ Ruth Lim \\ Massachusetts General Hospital \\ Barbara K. Winrich \\ Massachusetts General Hospital \\ Anca L. Grosu \\ Massachusetts General Hospital
}

See next page for additional authors

Follow this and additional works at: https://ro.uow.edu.au/eispapers

Part of the Engineering Commons, and the Science and Technology Studies Commons 


\title{
18F-FMISO PET/CT visualization of tumor hypoxia in patients with chordoma of the mobile and sacrococcygeal spine
}

\author{
Abstract \\ Abstract presented at the American Society for Radiation Oncology 56th Annual Meeting, ASTRO's 56th \\ Annual Meeting, San Francisco, United States, 14-17 September 2014 \\ Keywords \\ sacrococcygeal, mobile, chordoma, patients, hypoxia, tumor, spine, visualization, $18 \mathrm{f}$, ct, pet, fmiso \\ Disciplines \\ Engineering | Science and Technology Studies

\section{Publication Details} \\ Cheney, M. D., Chen, Y., Lim, R., Winrich, B. K., Grosu, A. L., Trofimov, A. V., Depauw, N., Shih, H. A., Schwab, \\ J. H., Hornicek, F. J. \& Delaney, T. F. (2014). 18F-FMISO PET/CT visualization of tumor hypoxia in patients \\ with chordoma of the mobile and sacrococcygeal spine. International Journal of Radiation: Oncology - \\ Biology - Physics, 90 (1, Suppl. 1), S753-S753.

\section{Authors} \\ Matthew D. Cheney, Yen-Lin Chen, Ruth Lim, Barbara K. Winrich, Anca L. Grosu, Alexei V. Trofimov, Nicolas \\ Depauw, Helen A. Shih, Joseph H. Schwab, Francis J. Hornicek, and Thomas F. Delaney
}




\title{
18F-FMISO PET/CT Visualization of Tumor Hypoxia in Patients With Chordoma of the Mobile and Sacrococcygeal Spine
}

\author{
M.D. Cheney,1 Y. Chen,2 R. Lim,2 B.K. Winrich,2 A.L. Grosu,2 A.V. Trofimov,2 N. Depauw,2,3 H.A. Shih,2 J.H. \\ Schwab,2 F.J. Hornicek, 2 and T.F. DeLaney2; \\ 1 Harvard Radiation Oncology Program, Boston, MA, 2Massachusetts General Hospital, Boston, MA, \\ 3University of Wollongong, Wollongong, Australia
}

Purpose/Objective(s): Local recurrence rates in reported series of chordoma patients following treatment with surgery \pm radiation therapy (RT) or definitive RT are high. Tumor hypoxia is associated with radioresistance and local recurrence in animal models and human patients. $\left[{ }^{18} \mathrm{~F}\right]$ fluoromisonidazole positron emission tomography/computed tomography (FMISO-PET/CT) has been used to visualize hypoxic sub-volumes (HSV) in skull base chordoma and the feasibility of its use in RT dose-escalation has been demonstrated in head and neck cancer. The feasibility of FMISOPET/CT use for detection of targetable HSVs in patients with chordoma of the mobile and sacrococcygeal spine is unknown and investigated in the current study.

Materials/Methods: A prospective, pilot study of 20 patients with primary or locally recurrent chordoma of the mobile or sacrococcygeal spine treated with proton or combined proton/photon RT \pm surgery was completed. FMISO-PET/CT was performed prior to RT and again after 19.8-34.2 GyRBE (relative biologic effectiveness). Gross tumor volumes were delineated and HSVs defined including all voxels with a standardized uptake value (SUV) $\geq$ 1.4 times the mean muscle SUV. The pre-specified threshold for FMISO-PET/CT feasibility was positive tracer uptake in 4/20 patients. Distributions of clinical characteristics and treatments received were compared between patients with and without HSVs. Treatment outcomes are reported.

Results: FMISO-PET/CT detected HSVs in 12 (60\%; 12/20) patients, 8 of which were of sufficient size ( $\geq 5 \mathrm{cc}$ ) to potentially allow for delivery of an intensity modulated proton therapy boost. Patients with HSVs had significantly larger gross tumor volumes (median = $410.0 \mathrm{cc}$ vs $63.4 \mathrm{cc} ; \mathrm{p}=0.02$ ) and were significantly more likely to have stage T2 tumors (5/12vs 0/8; $p=0.04$ ) compared to those without HSVs. After a median followup of 1.8 years (range: $0.2-4.4$ ), a local recurrence has yet to be observed. Three patients developed metastatic disease, 2 of whom had HSVs.

Conclusions: FMISO-PET/CT is feasible for detection of targetable HSVs within patients undergoing RT \pm surgery for treatment of chordoma of the mobile and sacrococcygeal spine. Further study of its application in hypoxia-directed, dose-escalated RT, particularly in patients at high risk for local recurrence, is warranted. 